\title{
An Aptameric Microfluidic System for Specific Purification, Enrichment, and Mass Spectrometric Detection of Biomolecules
}

\author{
Thai Huu Nguyen, Renjun Pei, Chunmei Qiu, Jingyue Ju, Milan Stojanovic, and Qiao Lin
}

\begin{abstract}
We present an innovative microfluidic system that accomplishes specific capture, enrichment, and isocratic elution of biomolecular analytes with coupling to label-free mass spectrometric detection. Analytes in a liquid phase are specifically captured and enriched via their affinity binding to aptamers, which are immobilized on microbeads packed inside a microchamber. Exploiting thermally induced reversible disruption of aptamer-analyte binding via on-chip temperature control with an integrated heater and temperature sensor, the captured analytes are released into the liquid phase and then isocratically eluted and transferred via a microfluidic flow gate for detection by matrix-assisted laser desorption/ionization mass spectrometry (MALDI-MS). The utility of the device is demonstrated using adenosine monophosphate (AMP) as a model analyte. Experimental results indicate that the device is capable of purifying and enriching the analyte from a sample mixed with nonspecific analytes and contaminated with salts. In addition, thermally induced analyte release is performed at modest temperatures $\left(45^{\circ} \mathrm{C}\right)$, and mass spectra obtained from MALDI-MS demonstrate successful detection of AMP at concentrations as low as $10 \mathrm{nM}$ following enrichment by consecutive infusion of a diluted sample.

[2009-0176]
\end{abstract}

Index Terms-Affinity binding, aptamer, enrichment, isocratic elution, mass spectrometry (MS), matrix-assisted laser desorption/ ionization (MALDI), purification.

\section{INTRODUCTION}

$\mathbf{M}$ ICROELECTROMECHANICAL systems (MEMS) have been widely applied in biomedical research by offering distinct advantages in footprint miniaturization, functionality integration, performance improvements, and potential cost reduction. As a particularly important advance

Manuscript received July 14, 2009; revised September 18, 2009. First published November 13, 2009; current version published December 1, 2009 This work was supported in part by the National Science Foundation under Grant CBET-0693274, Grant CBET-0854030, and Grant EIA-0324845, and in part by the Alternatives Research and Development Foundation. Subject Editor D. L. DeVoe.

T. H. Nguyen and Q. Lin are with the Department of Mechanical Engineering, Columbia University, New York, NY 10027 USA (e-mail: tn2144@ columbia.edu; qlin@ columbia.edu).

R. Pei and M. Stojanovic are with the Division of Clinical Pharmacology and Experimental Therapeutics, Department of Medicine, Columbia University, New York, NY 10032 USA (e-mail: rp2164@columbia.edu; mns18@ columbia.edu).

C. Qiu and J. Ju are with the Department of Chemical Engineering, Columbia University, New York, NY 10027 USA (e-mail: cq2122@columbia.edu; ju@ c2b2.columbia.edu).

Color versions of one or more of the figures in this paper are available online at http://ieeexplore.ieee.org.

Digital Object Identifier 10.1109/JMEMS.2009.2034392 in MEMS technology, microfluidics has been enabling a broad spectrum of miniaturized platforms for applications involving integrated and total analysis of biochemical samples. In such systems, various physical components and diverse bioanalytical functionalities can be integrated onto a single microchip, allowing the sensitive and reliable detection and investigation of biological phenomena in a controlled miniaturized environment with minimized sample and reagent consumption.

A major application of microfluidics technology has involved proteomics and genomics, enabling significant technological improvements to methods such as microarray gene sequencing, 2-D gel electrophoresis, radioimmunological detection assays, and mass spectrometry (MS) [1]. Among these methods, MS [2], including matrix-assisted laser desorption/ionization MS (MALDI-MS) [3], is widely used due to its label-free nature, excellent detection limits, and simplicity in data interpretation. In MALDI-MS, analytes are cocrystallized in an energyabsorbing matrix material on a support substrate (hereafter called a MALDI sample plate), which is then placed in a mass spectrometer. Laser irradiation causes ionization of the analytes, whose mass spectra can hence be obtained. The quality and efficacy of quantifiable MALDI-MS crucially depends on sample purity. Thus, samples typically need to be cleaned up to remove impurities such as salts, particulates, nonspecific molecules, and physiological tissue, while enriching analytes prior to MALDI-MS analysis. Solid-phase extraction (SPE) is one of the most commonly used sample cleanup techniques. During SPE, an analyte of interest within a fluid phase interacts through surface chemistry with a solid phase, allowing impurities remaining in the liquid phase to be removed by rinsing [4]. Typically, an organic solvent is used to elute and recover the analyte for further analysis. Compared with other sample preparation methods (e.g., electrokinetic sample stacking [5], liquid-liquid extraction [6], and dialysis [7]), SPE offers advantages, such as simultaneous sample cleanup and trace enrichment, as well as relatively short sample processing times.

Existing microfluidic SPE platforms for MALDI-MS primarily utilize physisorption capture of the target analyte by gels or membranes [8]. For example, based on commercial reversed-phase gels, microfabricated silicon chips have been used to enrich alcohol dehydrogenase [9], [10]. The captured protein molecules were eluted by addition of a polar solvent (acetonitrile) to change the polarity of the support surfaces. Alternatively, SPE microchips have also used ion-exchange 
supports, such as methacrylate-based gels [11], which utilize charged molecules on the retention media to interact with analytes [12], [13], and elution methods involving severe $\mathrm{pH}$ gradients. Using this approach, a microsystem consisting of a chip-integrated SPE array and a capillary force filling microdispenser performed reversed-phased purification and trace enrichment of digested proteins from fibroblast and epithelial cells [14]. Despite much progress, these microfluidic SPE devices suffer from two major limitations. First, as the devices rely on nonspecific interactions between the solid phase and analytes, the analyte enrichment is indiscriminate and often affected by contamination from impurities. Second, analyte elution in these devices requires the use of harsh acids or strong $\mathrm{pH}$ gradients, which may compromise the analyte and present other compatibility issues. These limitations are hindering microfluidic SPE devices from addressing the demands of MALDI-MS analysis, which increasingly requires the detection of analytes in complex samples containing impurities, such as cellular debris, nonspecific molecules, and salts [15], [16].

Aiming to address these limitations, this paper presents an integrated microfluidic SPE device for highly selective biomolecular purification and enrichment for enhanced MALDI-MS detection. We have recently shown that aptamer-functionalized microfluidic surfaces are capable of capturing an analyte and then releasing it by thermally interrupting the affinity binding with a modest temperature increase [17]. Building on that work, this paper demonstrates single-chip integration of aptamer-based specific biomolecular enrichment and manipulation for MALDI-MS detection, with a number of significant advances. First, in addition to using aptamers immobilized in a microchamber to capture and thermally release fluorescently labeled analytes [17], the device presented in this paper further demonstrates highly effective enrichment of analytes that are not labeled with any molecular groups. Second, in addition to integrated temperature control, the device allows integrated transfer of the enriched and released analytes to a MALDI sample plate by integrating the aptamer-based enrichment microchamber with sample manipulation microchannels and a surface tension-based flow gate. This, to our knowledge, is the first time that aptamer-based affinity extraction and MS are integrated using microfluidics technology. Finally, using the microfluidic device, extensive experimental data are obtained to demonstrate the promise of aptamer-based specific enrichment and label-free detection of biomolecules. This technological approach can ultimately be extended to create highly efficient array-based microfluidic platforms for high-throughput MALDI-MS analysis of biomolecular analytes in complex samples.

This paper is organized as follows: We first introduce the design and principle of the aptamer-based microfluidic enrichment system in Section II, whereas the experimental method is presented in Section III. In Section IV, we present results from systematic experimentation involving label-free detection of analytes at varying concentrations (Section IV-A), consecutive infusion of dilute analytes to enrich and enhance detection (Section IV-B), and finally demonstrate analyte purification from contaminants such as nonspecific analytes or salts (Sections IV-C and D). This paper concludes in Section V.

\section{PRinciple AND DESign}

Aptamers are oligonucleotides (usually 25-100 bases long) that recognize a broad class of analytes, such as small molecules [18], peptides [19], amino acids [20], proteins [21], viruses [22], and bacteria [23], via specific affinity interaction. Derived from ribonucleic (RNA) or deoxyribonucleic (DNA) acids, aptamers are isolated through an in vitro procedure called systematic evolution of ligands by exponential enrichment, whereby very large populations of random-sequence oligonucleotides (DNA or RNA libraries) are continuously screened against a target molecule until highly selective candidates are isolated and subsequently amplified [24]. Aptamers have been used in applications such as target validation [25], drug discovery [26], diagnostics [27], therapy [28], and in particular, analyte purification [29]. Employed in our microfluidic device, aptamers allow specific capture and thermally induced recovery of biomolecular analytes.

The microfluidic device consists of a microchamber packed with aptamer-functionalized microbeads for analyte capture and enrichment, a microheater and temperature sensor for thermally induced analyte release, and a passive flow gate for guiding the released analytes to a spotting outlet interfaced to a MALDI sample plate (Fig. 1). The microchamber is rounded in shape to reduce dead volume and bubble formation. Samples and reagents are introduced via the sample inlet and are directed to the waste outlet during purification and enrichment, or gated to the spotting outlet where the analyte is deposited onto a MALDI sample plate for MS detection [Fig. 1(a) and (b)]. Prior to that, the microchamber has been packed with aptamerfunctionalized microbeads through the bead inlet, which was sealed upon completion of bead packing. Narrow slits at the inlet and outlet of the microchamber are used to block the passage of microbeads, which are hence retained in the microchamber [Fig. 1(c)]. A serpentine resistive heater and temperature sensor are placed below the aptamer microchamber to promote efficient heating of the entire microchamber and accurate sensing at its center [Fig. 1(c)] while allowing closedloop temperature control for thermally induced release of the purified and enriched analyte.

The microfluidic flow gate, which directs the released analyte to the spotting outlet, exploits surface tension [Fig. 1(d)]. That is, a pressure difference exists at the air-liquid interface in a sudden narrowing of a microchannel with hydrophobic surfaces [30]. This pressure difference is provided by the Young-Laplace relationship and serves as a pressure barrier (i.e., critical pressure), which, only when exceeded, will allow the eluent (i.e., eluted sample) to enter the secondary channel and the spotting outlet. Note that the flow gate and the spotting outlet are placed close to the aptamer microchamber to reduce analyte dilution after release due to adsorption to the channel walls or diffusion into dead fluid volumes.

The microfluidic chip is realized with three sandwiched polymer layers [Fig. 1(b)]. The bottom layer incorporates the inlets, flow gate, and waste outlet. To reduce bubble entrapment or dead volumes during sample spotting, the middle layer provides an air vent connected to the spotting outlet. A meniscus forms in the air vent channel, through which trapped gas bubbles in the 


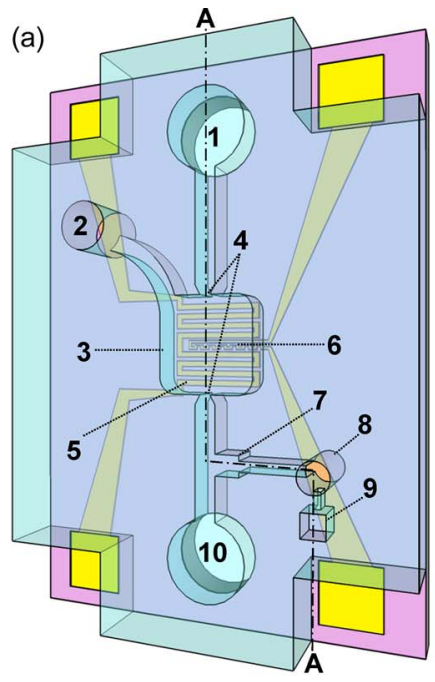

1. Sample Inlet

2. Bead Inlet

3. Aptamer Microchamber

4. Narrow Slits

5. Heater

6. Temperature Sensor

7. Flow Gate

8. Spotting Outlet

9. Air Vent

10. Waste Outlet

11. Microbeads
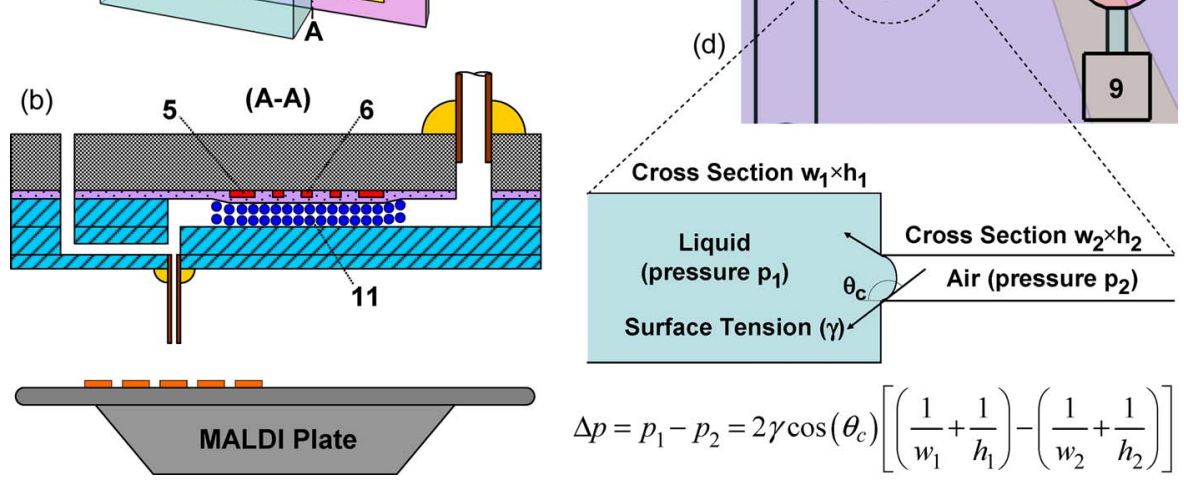

Fig. 1. (a) Schematic of the microfluidic purification and enrichment device. (b) Cross-sectional view along line A-A, as shown in (a). The coupling of the device to a MALDI sample plate is also shown. (c) A close-up top view of the aptamer microchamber area. (d) A close-up top view of the flow gate and analyte spotting area, with the flow gating principle illustrated. Fluid flow across the sudden contraction in channel cross-sectional area will occur only when the driving pressure exceeds the pressure barrier $\Delta p$ at the air-liquid interface in the hydrophobic channel.

spotting outlet can be eliminated. Additionally, the middle layer encapsulates and seals the microfluidic network formed in the bottom layer. Finally, the top layer defines the spotting outlet and houses the air vent channel. To interface the device to the sample plate, the device incorporates a glass capillary that is fitted to the opening of the microchip spotting outlet. For example, samples are ejected from the capillary tip by hydrodynamic force and allowed to crystallize before MS analysis.

During operation, an aqueous sample containing a biomolecular analyte intermixed with nontarget molecules is introduced to the aptamer-functionalized beads within the microchamber and thus is extracted by the aptamer. This occurs at a suitable (e.g., room) temperature so that the aptamer specifically captures the analyte from the liquid phase, whereas the impurities are flushed from the system through the waste outlet. The foregoing sequence is repeated in a discrete (consecutive infusion of dilute sample) fashion to adequately purify and enrich the analyte, if necessary. Subsequently, the aptamer-analyte binding is reversibly disrupted by altering the temperature of the solid support such that the enriched analyte is released into a plug of pure aqueous buffer, or MALDI matrix solution, which is then eluted through the passive flow gate (that is activated by closing the waste outlet) onto a MALDI sample plate for MS detection. Thus, this thermally induced analyte release method allows isocratic elution, i.e., elution within a single aqueous mobile phase, and eliminates the use of potentially harsh or harmful reagents. Additionally, returning the temperature to the initial state allows the aptamer to revert to its initial functional structure, i.e., the aptamer-functionalized surfaces are regenerated.

To demonstrate the device operation, we utilize a model binding system consisting of adenosine monophosphate (AMP), which is recognized by an RNA aptamer derived for adenosine triphosphate (ATP-aptamer). AMP is an important nucleotide involved in metabolic processes and is also required physiologically to prevent AMP deaminase, a condition causing premature muscle fatigue during exercise [31]. AMP is captured by ATPaptamer through an induced 11-base loop flanked by doublestranded RNA, which forms an affinity binding epitope for the small molecule.

\section{EXPERIMENTAL}

\section{A. Materials and Instrumentation}

The adenosine triphosphate aptamer (ATP-aptamer with sequence: 5'-GGGUUGGGAAGAAACUGUGGCACUUCGGUGCCAGCAACCC- $3^{\prime}$ ), with a $5^{\prime}$-end functionalized with biotin, was acquired through Integrated DNA Technologies. As MALDI-MS is inherently sensitive to salt impurities, DNAgrade water (sterile RNase/Protease-free water, Fisher) was used to prepare ATP-aptamer, AMP, cytidine, uridine, and guanosine triphosphate (CTP, UTP, and GTP, respectively) 
(a)

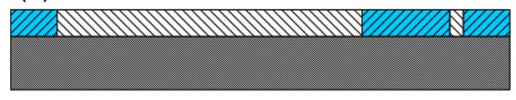

(b)

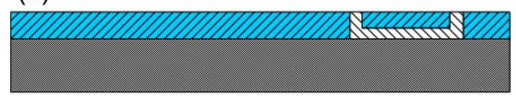

(c) (d)

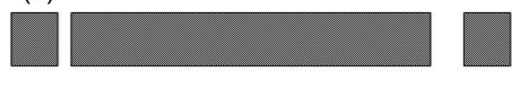

(e)

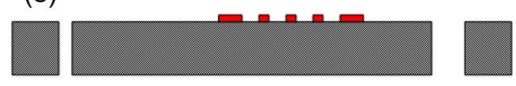

(f)

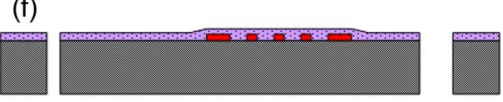

(g)
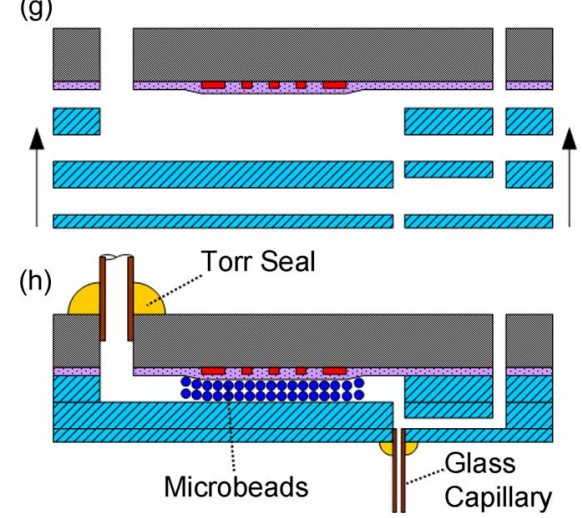

Fig. 2. Device fabrication flow as seen from cross section A-A in Fig. 1(a). (a)-(c) SU-8 patterning followed by subsequent PDMS prepolymer casting to form microfluidic layers. (d) Glass substrate drilled for fluidic interconnects. (e) Thermal evaporation and lift-off patterning of Cr/Au bilayer. (f) PECVD deposition of $\mathrm{SiO}_{2}$ passivation layer. (g) Microfluidic structural layers aligned and permanently bonded to the glass substrate. (h) Packaged chip with tubing.

samples in addition to being used for all wash steps. All sample nucleotides were purchased from Sigma. An aqueous buffer solution ( $\mathrm{pH}$ 7.4) was prepared with a mixture of water, Tris- $\mathrm{HCl}(20 \mathrm{mM}), \mathrm{NaCl}(140 \mathrm{mM}), \mathrm{KCl}(5 \mathrm{mM})$, and $\mathrm{MgCl}_{2}$ (5 mM). The MALDI matrix solution (THAP), with compounds acquired through Sigma, was prepared from 2,4,6trihydroxy-acetophenone (2,4,6-THAP), 2,3,4-THAP, and diammonium citrate at $0.1,0.05$, and $0.075 \mathrm{M}$ concentrations, respectively, in a $3: 5(\mathrm{v}: \mathrm{v})$ mixture of acetonitrile/water. Fluorescein powder was acquired from Sigma-Aldrich and solvated in DNA ethanol for solution preparation. UltraLink streptavidin-coated bis-acrylamide/azlactone beads were acquired from Pierce and used to immobilize the ATP-aptamer via a biotin-streptavidin link. Microfabrication materials, including SU-8 2025 and 2100, Remover PG, Sylgard 184 polydimethylsiloxane (PDMS), Torr Seal epoxy, polyethylene (PE) film (76 $\mu \mathrm{m}$ thick), and microscope grade glass slides $(25 \mathrm{~mm} \times$ $75 \mathrm{~mm}$ ), were purchased from MicroChem, Dow Corning, Varian, 3M, and Fisher, respectively. A mercury-vaporinduced epi-fluorescence microscope (Nikon Eclipse TE300) coupled with a charge-coupled device camera (Micrometrics Accu•Scope 190CU) was used for fluorescence experiments. A dc power supply and a proportional-integral-derivativecontrolled LabVIEW program were used in parallel to control temperature during thermally initiated analyte release from aptamer molecules. Finally, a New Era model NE-1000 syringe pump was used for sample introduction, whereas a VoyagerDE time-of-flight mass spectrometer (Applied Biosystems) was used for mass analysis.

\section{B. Device Fabrication}

The device was fabricated from PDMS and bonded on a glass substrate using standard soft lithography techniques (Fig. 2). SU-8 masters for each microfluidic layer (100, 80, and $100 \mu \mathrm{m}$ consecutively from the substrate surface) were first generated on silicon wafers. A PDMS prepolymer solution was mixed $(10: 1 / \mathrm{v}: \mathrm{v})$ and then poured onto individual masters. A PE film (preferentially coated with an adhesive on one side) was subsequently layered over the prepolymer mixture, allowing surface tension forces to make intimate contact between the prepolymer and PE film. The master/prepolymer/transparency stack was then clamped within a through-hole PDMS sandwich assembly and cured for $45-60 \mathrm{~min}$ at $60^{\circ} \mathrm{C}$. This produced thin PDMS microfluidic layers (of equal thickness to their respective SU-8 masters) that can be peeled off from the masters, via the PE films, for bonding to the glass substrate. Meanwhile, glass substrates were diced $(25 \mathrm{~mm} \times 30 \mathrm{~mm})$ and drilled to create the inlets and outlets. Subsequently, Cr/Au (5/100 nm) thin films were deposited and patterned on the substrates via thermal evaporation and then passivated with $\mathrm{SiO}_{2}$ using plasma-enhanced chemical vapor deposition (PECVD), realizing the microheater and temperature sensor. Following an $\mathrm{O}_{2}$ plasma treatment of each bonding interface (125 mtorr, $85 \mathrm{~W}$, and $12 \mathrm{~s}$ ), all three PDMS layers and the glass substrate were aligned and permanently bonded. A glass capillary tube $(5 \mathrm{~mm} \times 0.5 \mathrm{~mm}$ I.D.) was then inserted into the spotting outlet and fastened with Torr Seal. Finally, microbeads were packed into the microchamber and the fluidic ports were sealed. A packaged device is shown in Fig. 3. 


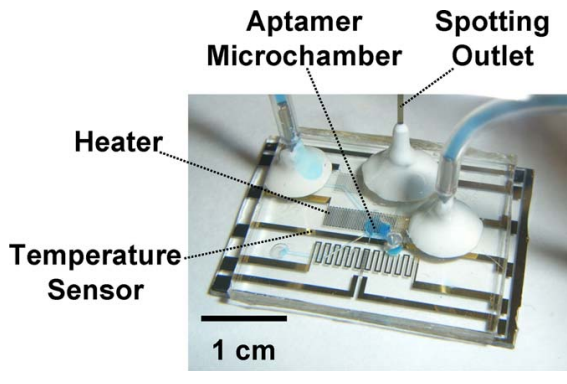

Fig. 3. Photograph of a packaged device.

\section{Experimental Protocols}

The microfluidic device (Fig. 3) used in our experiment had a microchamber with overall dimensions of $3 \mathrm{~mm} \times 3 \mathrm{~mm} \times$ $180 \mu \mathrm{m}$ and an effective volume of $1.62 \mu \mathrm{L}$. We utilized microbeads with diameters ranging from 50 to $80 \mu \mathrm{m}$, which were retained in the microchamber by narrow slits $(40 \mu \mathrm{m}$ in width). This diameter range was used to facilitate the proofof-concept and can considerably be decreased by reducing the bead-retaining slit width as well as the microchamber volume. The fabricated microheater had a resistance of $500 \Omega$, whereas the microtemperature sensor had a resistance of $27.5 \Omega$ with a temperature coefficient of resistance of $2.65 \times 10^{-3} /{ }^{\circ} \mathrm{C}$. They were used in conjunction with off-chip programming to achieve closed-loop chip temperature control.

The device was first primed with a water wash $(10 \mu \mathrm{l} / \mathrm{min}$, $10 \mathrm{~min}$ ). All of the following experimental washing and loading schemes were identical. These parameters were specified based on the microchamber size $(1.62 \mu \mathrm{l})$, which was created such that with the microbeads packed at an expected $63.5 \%$ efficiency a fluid volume of slightly over $1 \mu \mathrm{L}(1.02 \mu \mathrm{L})$ can be attained. This is within the common volume range for sample spotting used in MALDI-MS analysis $(0.5-2 \mu 1)$. Initially, a $10-\mu \mathrm{M}$ ATP-aptamer sample $(10 \mu \mathrm{l})$ was loaded into the microchamber and allowed to incubate with the streptavidinfunctionalized bead bed (40 min). After subsequent washing, the device was ready for sample introduction. In parallel, a device not functionalized with ATP-aptamer (control device) was used to process control samples of AMP, CTP, UTP, and GTP ( $1 \mu \mathrm{l}$; similar for all sample/matrix volumes in the following experiments), which were prepared at $1 \mu \mathrm{M}$ each. The operational principle described in Section II was used. Manually pipetted AMP, CTP, UTP, and GTP samples at 1- $\mu \mathrm{M}$ concentrations were deposited and analyzed to obtain reference spectrums. The separate data sets were compared to reveal the sample loss incurred within the device during device operation.

Fluorescein solution was used to characterize the operation of the flow gate. The solution was first flowed at a flow rate of $10 \mu \mathrm{l} / \mathrm{min}$ through the microchamber and the waste outlet below the critical pressure of the flow gate. To activate the passive flow gate, the waste outlet was closed while maintaining a constant flow rate. This increased the pressure in the flow stream adjacent to the flow gate to eventually overcome its critical pressure and activate the gate. A $10 \times$ microscope objective was focused on the flow gating area and adjoining channels during experimentation.
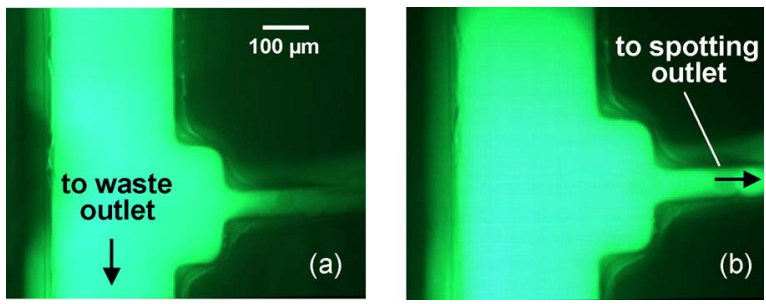

Fig. 4. Demonstration of flow gate operation. (a) Transfer of fluorescein solution to the waste outlet while blocking the spotting outlet (notice that fluorescein enters the channel leading to the spotting outlet only by slow diffusion and not bulk fluid flow). (b) Transfer of a fluorescein solution to the spotting outlet.

The extraction and purification experiments using the microdevice were performed as follows (refer to Fig. 1): First, 10-nM, 100-nM, and 1- $\mu \mathrm{M}$ AMP samples were separately loaded via the sample inlet into the aptamer microchamber. A rinse followed to rid nonspecific compounds. AMP was then released from the aptamer by raising the microchamber temperature to $45^{\circ} \mathrm{C}$ using a closed-loop thermal control with the microheater and temperature sensor. Meanwhile, a matrix sample plug was introduced to mix with the released analyte, which was then transferred from the aptamer microchamber, through the flow gate, to the spotting outlet and deposited onto the MALDI plate for subsequent mass spectrometric analysis. Similarly, for experiments involving specific purification of AMP from model impurities, a solution of AMP $(100 \mathrm{nM})$, CTP $(1 \mu \mathrm{M})$, UTP $(1 \mu \mathrm{M})$, and GTP $(1 \mu \mathrm{M})$ was loaded into the microchamber. After a short incubation (3 min), as described in our previous work [17], the impurity molecules were washed from the microchamber, and the matrix was introduced. Heat was applied, while the passive flow gate was activated, to release the molecules currently on the aptamer and deposit them onto the MALDI plate for analysis. For enrichment and enhanced detection of AMP, a multiple infusion scheme was used. The aptamer microchamber was consecutively loaded with 10-nM infusions of AMP sample. Each infusion was incubated (3 $\mathrm{min}$ ) and followed by a rinse. Upon apparent saturation of the aptamer with AMP, the microchamber was heated to release the analytes into a matrix plug, which was deposited for analysis.

\section{RESUlts AND Discussion}

To ensure the validity of our higher-level data, properties of the surface-tension-based flow gate (Fig. 4), in addition to the absorption/adsorption characteristics of the microfluidic structure, were obtained. At a steady-flow rate, the pressure difference imparted by the passive flow gate impinged fluid access to the spotting outlet. When the waste outlet was open and at flow rates below $50 \mu \mathrm{l} / \mathrm{min}$ (e.g., $10 \mu \mathrm{l} / \mathrm{min}$ ), fluid flow bypassed the flow gate since the hydrodynamic pressure driving flow ( $\sim 686 \mathrm{~Pa}$ [32]) was smaller than the critical pressure of the flow gate [Fig. 4(a)]. To direct flow to the MALDI plate, the pressure drop between the sample inlets to the waste outlet must be greater than the flow gate's critical pressure (i.e., above $3.154 \mathrm{kPa}$ ). This was accomplished by plugging the waste outlet using an external valve and maintaining a constant flow rate, 
which allowed fluorescein solution to enter the channel leading to the spotting outlet [Fig. 4(b)]. Additionally, analyte loss during fluidic transfer from the microchamber to the spotting outlet seemed negligible, since the data obtained from samples spotted using the control device consistently matched the reference samples, which were manually pipetted onto the sample plate. For conciseness, these data were not shown, but its significance is noted for further MALDI experimentation.

\section{A. Extraction, Thermally Induced Release, and MALDI-MS of AMP}

To demonstrate the ability to extract and detect AMP by MALDI-MS using our device, we first infused discrete samples of varying concentrations of AMP $(10 \mathrm{nM}, 100 \mathrm{nM}$, and $1.0 \mu \mathrm{M})$ into the aptamer microchamber. After interaction with the aptamer-functionalized beads, the AMP molecules were released and transferred to the spotting outlet and finally deposited onto a MALDI-MS plate. Mass analysis followed (Fig. 5). For attempted extraction and detection of samples with concentration at $10 \mathrm{nM}$ [Fig. 5(a)], no appreciable signal could be obtained above the noise level. In fact, only mass peaks corresponding to the THAP matrix were present, such as $339.44,392.45,468.23$, and $502.05 \mathrm{Da} / \mathrm{z}$. These mass spectral peaks were easily distinguishable by the mass spectrometer (Voyager DE), which can reliably resolve mass differences down to ca. $1 \mathrm{Da}$ over a mass range of 0-1000 Da. The mass spectrum of a spot obtained from a 100-nM AMP solution [Fig. 5(b)] showed a distinctive mass peak of $348.11 \mathrm{Da} / \mathrm{z}$, which corresponds to AMP (established value: $348.22 \mathrm{Da} / \mathrm{z}^{1}$ ) and indicates that the potential detection limit of our device lies between a range of 10-100 nM. Since the AMP concentration was still relatively low for this case, the magnitude of its peak was comparable to several peaks from the MALDI matrix (393.99 and 468.65 Da/z). Nonetheless, this detection sensitivity is ca. one order lower than physiologically relevant AMP levels in plasma [33]. In addition, a mass spectrum obtained from a 1.0- $\mu \mathrm{M}$ AMP solution [Fig. 5(c)] improved the analyteto-matrix peak contrast. In this case, the AMP peak clearly dominated the matrix peak amplitudes and indicated a nonlinear dependence of detection signal to infused sample concentration. Furthermore, this result suggested that enriching undetectable dilute samples can improve the analyte detection limit.

\section{B. Enhancement of AMP Detection by Enrichment of a Dilute Solution}

For high-sensitivity MALDI-MS, analyte sample conditioning and enrichment may be both useful and necessary to improve the detection signal. We investigated the ability of our

\footnotetext{
${ }^{1}$ Although mass spectrometry is a precise detection technique, various fluctuations in instrument settings (e.g., electromagnetic field strength, detector vibrations, and laser intensity) will cause expected $\mathrm{m} / \mathrm{z}$ values of a substance to vary slightly. Hence, the molecular ion peak in $\mathrm{m} / \mathrm{z}$ for AMP in this work will not always be exactly at 348.22 and would rather deviate slightly. Such slight deviations do not affect molecular identification and are generally accepted for mass spectrometry.
}
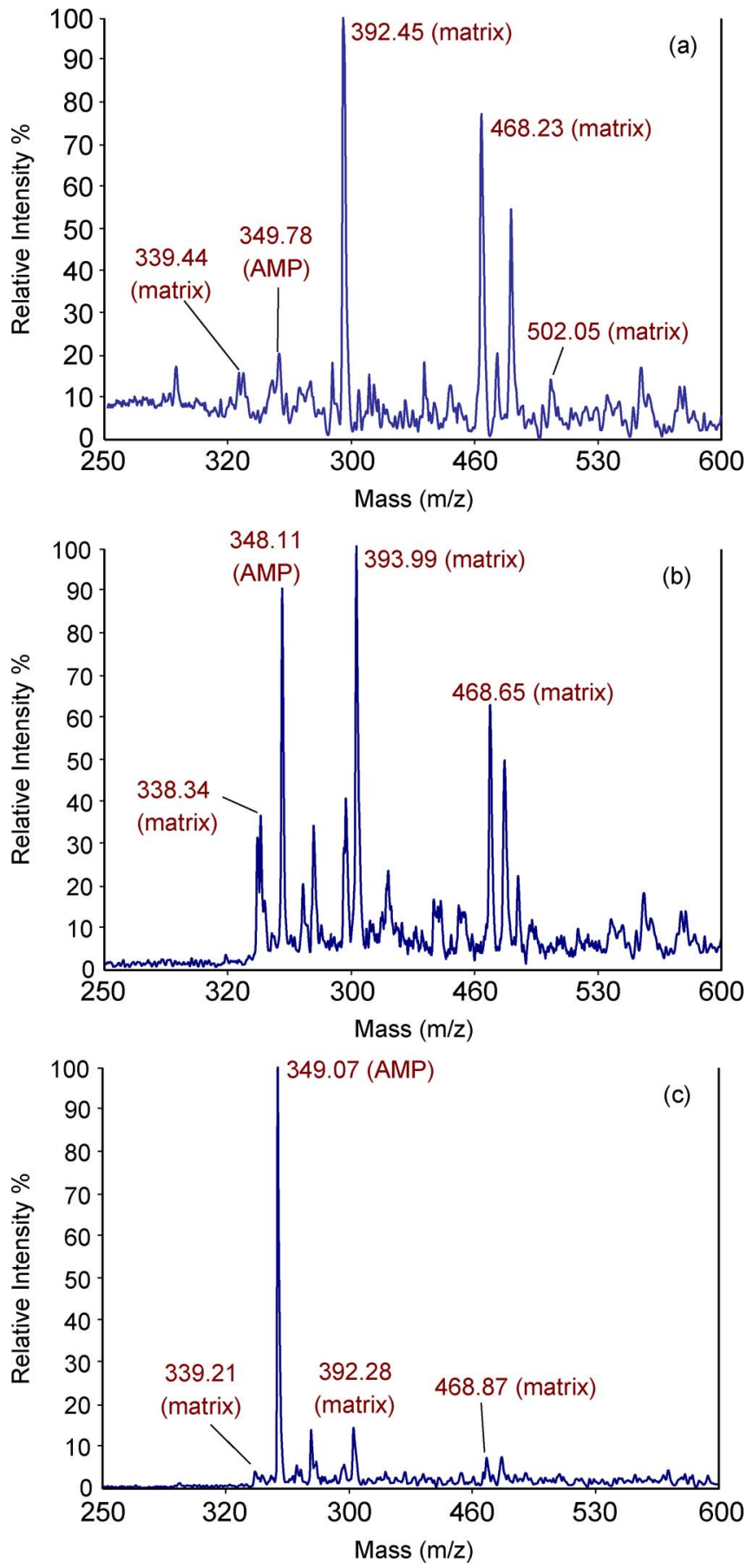

Fig. 5. MALDI-MS detection of varying concentrations of AMP in a pure water solution using an ATP-aptamer-functionalized microchip coupled to a MALDI sample plate: (a) $10 \mathrm{nM}$, (b) $100 \mathrm{nM}$, and (c) $1 \mu \mathrm{M}$.

device to enhance a sample of AMP $(10 \mathrm{nM})$, which was previously established to be undetectable in Section IV-A, by loading the dilute AMP sample into the aptamer microchamber multiple times to saturate the analyte on the aptamer before release and mass spectrometric analysis. A dilute sample concentration was chosen to be much lower than $100 \mathrm{nM}$ to highlight the detection enhancement due to this method of enrichment. We infused 25 consecutive dilute AMP samples into the aptamer microchamber, released the captured AMP with heat into a pure matrix solution, and transferred the concentrated plug to the 

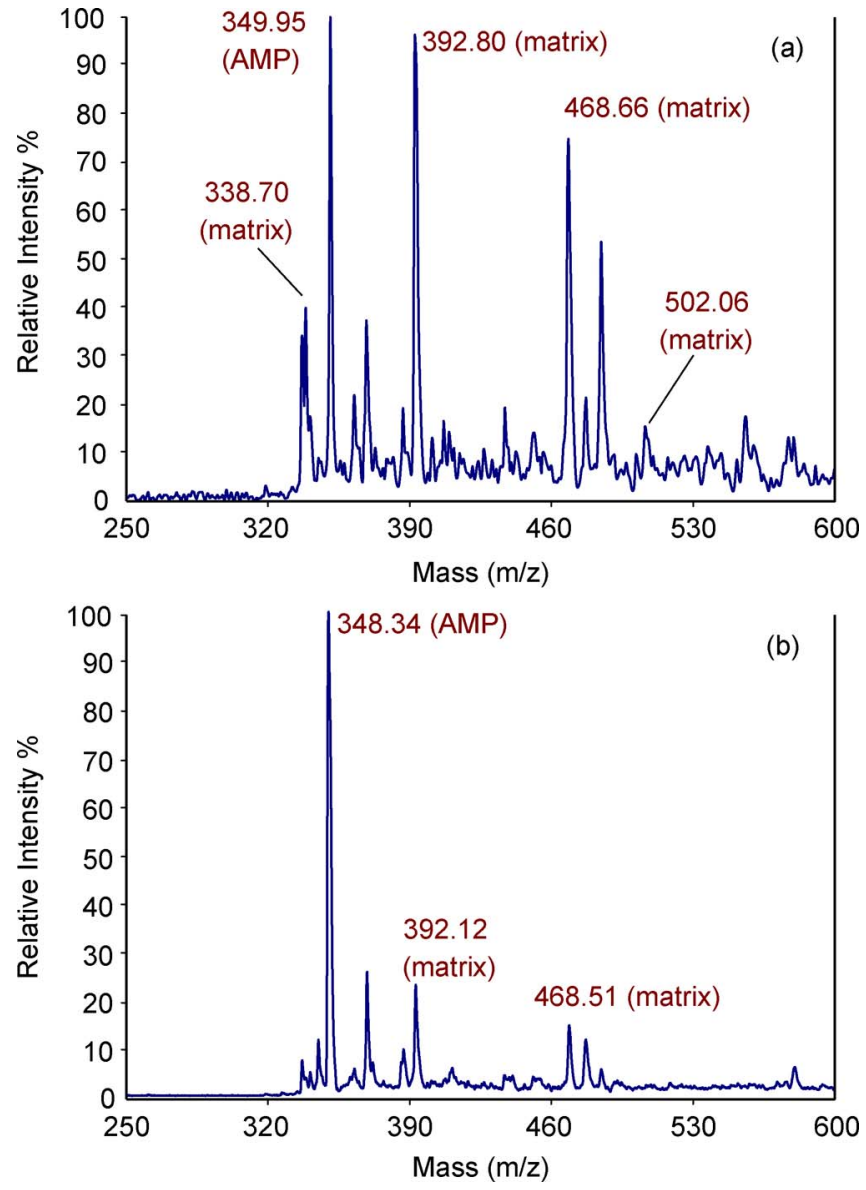

Fig. 6. Discrete concentration after (a) 25 and (b) 250 infusions of a 10-nM AMP sample revealing detection enhancement by aptamer-based enrichment.

spotting outlet. A spectrum was obtained from the resulting sample spot [Fig. 6(a)]. We noticed an AMP-to-matrix peak ratio slightly higher than that seen in Fig. 5(b), demonstrating the successful concentration of AMP by $\sim 10 \times$. This initial result did not reveal an intuitively anticipated steadily increasing relationship between infusion number and concentration factor [i.e., we did not see an AMP-to-matrix peak ratio roughly corresponding to $2 \times$ that in Fig. 5(b)]. This is likely due to the fact that binding in aptamer receptor systems is quite nonlinear in nature and significantly depends on the equilibrium kinetics of surface interactions for each particular system. However, this result indeed establishes the capabilities of our microchip for enhancing the detection of low concentration analytes so as to facilitate label-free detection by MALDI-MS.

To emphasize the capacity of this approach, more consecutive infusions of dilute (10 nM) AMP solution were performed to achieve a maximum enrichment factor for our device. A maximum of 250 infusions were performed [Fig. 6(b)]. Following the final infusion, a sample spot was obtained and analyzed with MALDI-MS, similar to the foregoing protocol with 25 infusions. Note that the AMP peak dominated those of matrix peaks, and the AMP-to-matrix peak ratio was comparable with that in Fig. 5(c). This suggested an AMP analyte enrichment factor of nearly $100 \times$. This is a significant concentration factor, which is comparable with that seen in reverse-phase SPE systems [1], but with the advantage of higher specificity and affinity imparted by aptamers. Moreover, by using our enrichment protocol, the detection limit of the device to AMP was improved by an order of magnitude (compared with the results demonstrated in Section IV-A) and allowed AMP detection at concentrations of two orders below physiologically relevant levels. An interesting point to highlight is that we stopped AMP sample infusions after 250 since satisfactory signal enhancement was achieved at this time, not because of actual saturation of the analyte on the aptamer microbeads. The signal gain achieved in Fig. 6(b) is merely the apparent signal enhancement, since the potential for even larger enrichment factors and higher signal gain is possible with our microchip [34]. It is also significant to mention that although the repeatability of our experiments is not explicitly gleaned from the presented data (due to the limits of presenting spectroscopic data), the microchip was easily regenerated (using thermal stimulation of the aptamer-functionalized beads) to allow reuse and repeated functionality [17].

\section{Purification and Enrichment of AMP in the Presence of Model Impurities}

Purification of analytes is a valuable tool for selectively controlling analytes in biochemical applications. We selectively isolated and enhanced the signal of AMP (100 nM) from a homogeneous solution among CTP, UTP, and GTP (model nonspecific analytes at $1.0 \mu \mathrm{M}$ each) by loading the sample into the microchamber and subsequently washing to isolate AMP on the aptamer-functionalized beads. To emphasize the power of aptamer purification, the ratio of AMP to nonspecific impurity analytes was reduced $(1: 10)$ to more closely mimic a common practical situation in which a target analyte may be in unfavorable disproportion to nontarget analytes. A deposited sample spot was similarly obtained with all the previous protocols. The control device was used to establish a reference spectrum for an unclean sample. Both samples were compared to delineate the effectiveness of aptamer-based sample cleanup prior to MALDI-MS (Fig. 7). For the control sample [Fig. 7(a)], the ratio of AMP to matrix was comparable with that seen in Fig. 5(b), where only AMP was present in the solution. However, the nontarget peaks corresponding to the model impurities were observed: CTP (480.01), UTP (484.51), and GTP (523.74) Da/z, which appear to have an adverse effect on the signal quality. Although the impurity nucleotide peaks do not prevent the identification of AMP, the experiment was intended to represent practical systems where biological impurities can severely degrade the analyte signal quality. The control sample signal was unlike that obtained utilizing the aptamer-functionalized microchip, where cleaning of the AMP sample through extraction and purification was possible [Fig. 7(b)]. There was a significant reduction of all impurity peaks (particularly that of CTP) while improving upon the AMP signal. Although CTP, UTP, and GTP were still present, their peaks appeared significantly lower than the AMP peak for this case, suggesting the amount of nonspecific binding was negligible to the AMP-specific aptamer. This is important since nonspecific binding can severely degrade MALDI-MS detection for practical applications. 

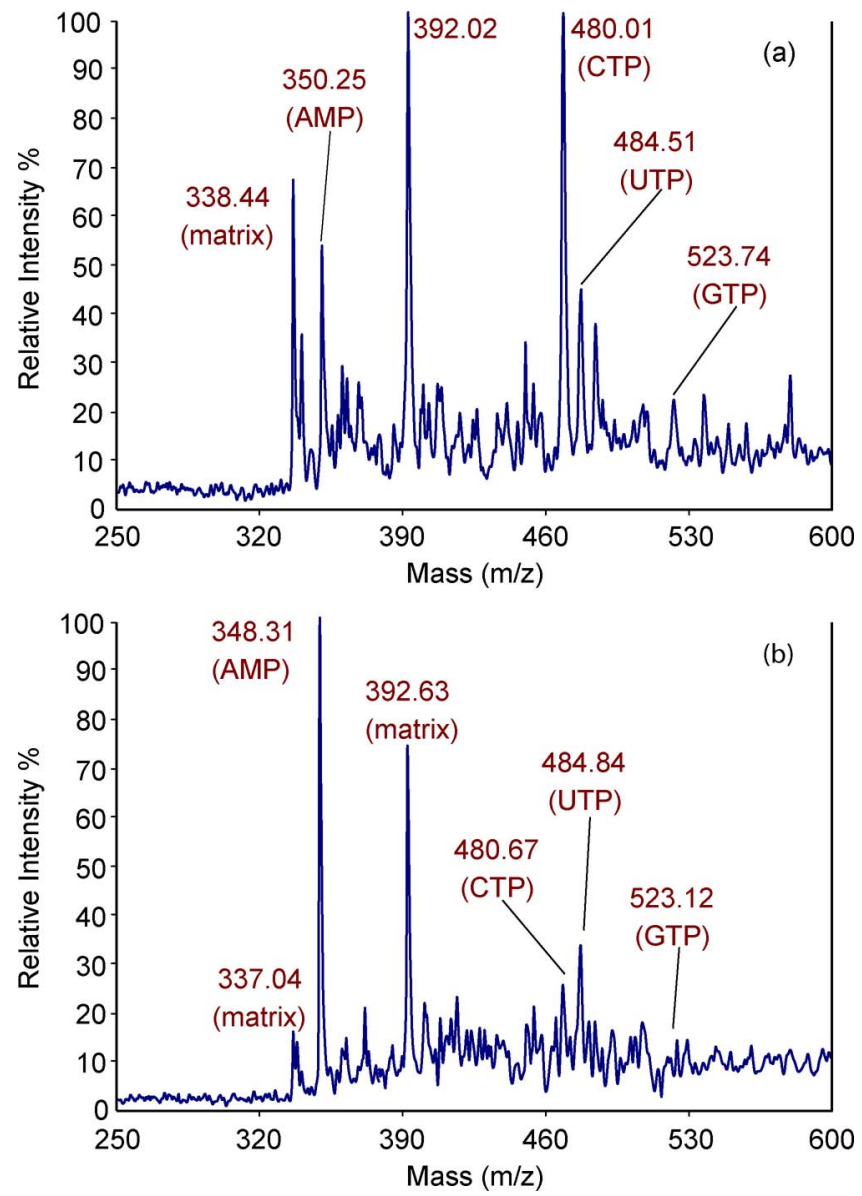

Fig. 7. Demonstration of the sample cleanup of a model analyte system before MALDI-MS detection. (a) MALDI spectrum of AMP (100 nM) in the presence of model impurity analytes (CTP, UTP, and GTP, all $1.0 \mu \mathrm{M}$ ). (b) MALDI spectrum of AMP in the presence of model impurities after cleanup using the aptamer-functionalized microchip device.

\section{Purification and Enrichment of AMP in the Presence of Salt Contaminants}

Along with potential interference from nonspecific analytes, MALDI analysis may also be hindered by contamination of salts present in both conditioned and physiological solutions [35]. Since it is inevitable that a particular analyte will be solvated within a solution stemming from one of these sources, the need to address this type of contamination in analytical samples is significant before performing MALDI-MS. We established the ability of our microdevice to selectively isolate AMP and enhance its detection from a buffer solution contaminated with common $\mathrm{pH}$ altering salts (e.g., Tris- $\mathrm{HCl}$, $\mathrm{NaCl}, \mathrm{KCl}$, and $\mathrm{MgCl}_{2}$ ). Primarily, these compounds degrade the baseline generated for a given MALDI spectrum (e.g., the baseline is translated considerably above $0 \mathrm{Da} / \mathrm{z}$ ), which can alter the relative intensities of significant analyte peaks as well as produce unwanted noise [36]. A 100-nM AMP sample in buffer solution was initially desalinated by infusing the sample into the aptamer microchamber to allow specific interaction of the AMP to ATP-aptamer. Flushing the buffer solution through the waste outlet followed by a short wash step allowed the analyte to be purified. This was followed with an infusion of a pure matrix plug and simultaneously initiating
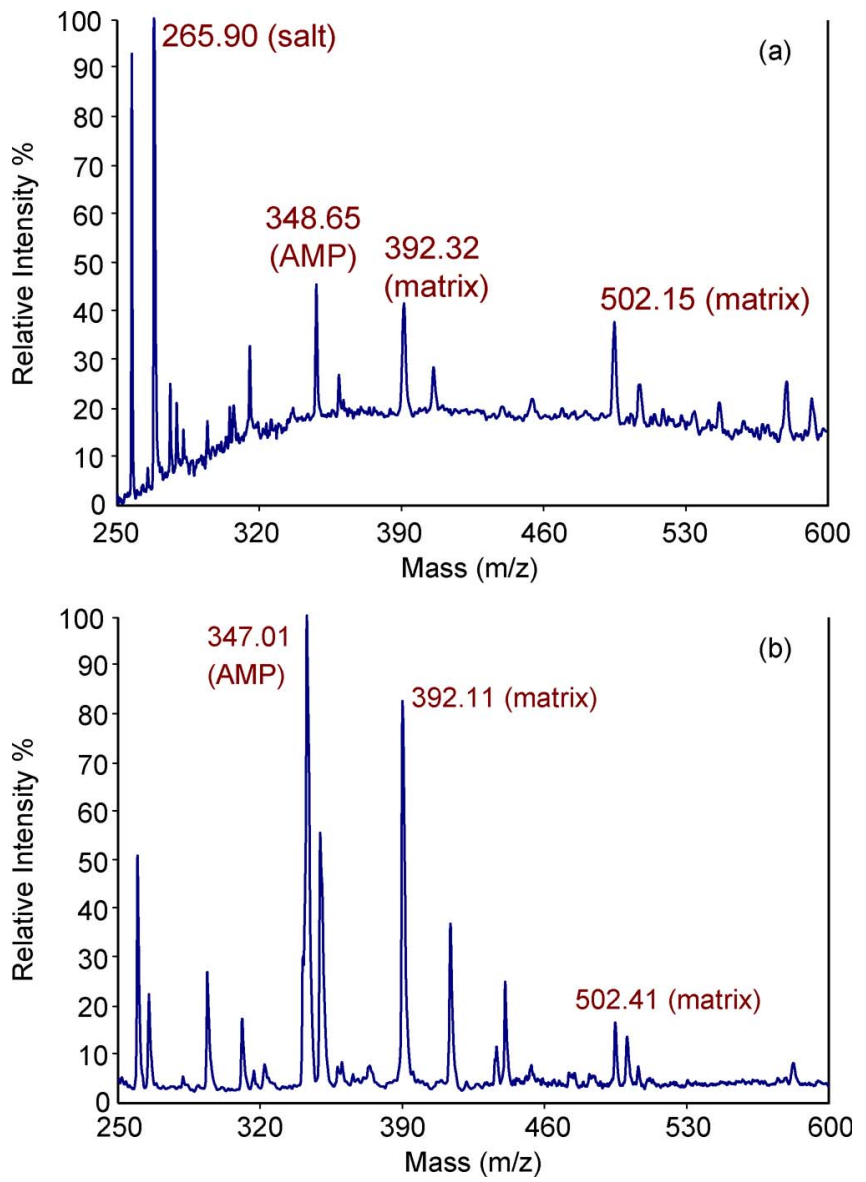

Fig. 8. Purifying AMP (100 nM) from a salt-contaminated buffer solution for enhanced MALDI-MS detection. (a) Spectrum of sample prior to purification using the microchip. (b) Spectrum obtained after sample purification using aptamer-functionalized microchip.

thermal release, sample transfer to the spotting outlet, and deposition of the analyte (similar to all previous protocols). The control microchip was used similar to that described in Section IV-C to establish a reference spectrum for the saltladen sample. The spectrums were compared to reveal the effective desalting capability of the device (Fig. 8). The control sample spectrum [Fig. 8(a)] revealed characteristic properties of a salt-contaminated sample. Particularly, the baseline of the spectrum was raised significantly above $0 \%$ intensity, altering the relative ratios of significant mass peaks. The AMP mass peak was barely registered above the baseline and noise peaks (265.90 Da/z) due to buffer salts, which dominated instead. After using the aptamer-functionalized chip for the same AMP sample, we observed a reduction of the baseline to near $0 \mathrm{Da} / \mathrm{z}$ in addition to an enhanced AMP mass peak signal [Fig. 8(b)]. All impurity and salt peaks (e.g., $265 \mathrm{Da} / \mathrm{z}$ ) were significantly reduced, which highlights the benefits of this microchip for desalination sample conditioning before MALDI-MS. This reduction was in good agreement with existing MEMS MALDIMS devices that indicate the necessity of desalination [37].

\section{CONCLUSION}

MEMS and microfluidic technology have entered many areas of biomedical research, including proteomics and genomics. 
In particular, it has been applied for improving MALDI-MS detection of analytes, which is a common analytical technique used in proteomics and genomics. This is generally due to impurities (e.g., nonspecific analytes and $\mathrm{pH}$ altering salt compounds) affecting the resulting analyte signal by degrading the signal strength. This paper has presented a novel microfluidic system that accomplishes specific capture, enrichment, and isocratic elution of biomolecular analytes for integrated labelfree MALDI-MS detection. Our approach offers distinct advantages over existing systems, particularly platforms utilizing SPE. For example, the microchip is capable of specific analyte purification and enrichment, low-temperature analyte release and device surface regeneration (which allows device reuse as needed), and a simplified design and fabrication process. In addition, the device is efficiently coupled to a MALDI sample plate, which allows offline label-free detection. We showed practical application of this device by demonstrating purification and enhanced enrichment (by $\sim 100 \times$ ) detection of AMP at trace levels and from contaminated solutions. Future research will investigate the suitability of our system for practical diagnostic and therapeutic applications.

\section{ACKNOWLEDGMENT}

The authors would like to thank Dr. J. Edwards at the Columbia Genomics Center for advice with MALDI analytical protocols and insight with data interpretation.

\section{REFERENCES}

[1] N. Lion, T. C. Rohner, L. Dayon, I. L. Arnaud, E. Damoc, N. Youhnovski, Z. Y. Wu, C. Roussel, J. Josserand, H. Jensen, J. S. Rossier, M. Przybylski, and H. H. Girault, "Microfluidic systems in proteomics," Electrophoresis, vol. 24 , no. 21 , pp. $3533-3562$, Nov. 2003.

[2] J. J. Li, T. LeRiche, T. L. Tremblay, C. Wang, E. Bonneil, D. J. Harrison, and P. Thibault, "Application of microfluidic devices to proteomics research-Identification of trace-level protein digests and affinity capture of target peptides," Mol. Cell. Proteomics, vol. 1, no. 2, pp. $157-168$, Feb. 2002

[3] M. Vestal and K. Hayden, "High performance MALDI-TOF mass spectrometry for proteomics," Int. J. Mass Spectrom., vol. 268, no. 2/3, pp. 8392, Dec. 2007.

[4] N. J. K. Simpson, Solid-Phase Extraction, 1st ed. New York: Marcel Dekker, 2000

[5] M. J. Gong, K. R. Wehmeyer, P. A. Limbach, F. Arias, and W. R. Heineman, "On-line sample preconcentration using field-amplified stacking injection in microchip capillary electrophoresis," Anal. Chem., vol. 78, no. 11, pp. 3730-3737, Jun. 2006.

[6] R. N. Wu, F. L. Han, J. Shang, H. Hu, and L. Han, "Analysis of patulin in apple products by liquid-liquid extraction, solid phase extraction and matrix solid-phase dispersion methods: A comparative study," Eur. Food Res. Technol., vol. 228, no. 6, pp. 1009-1014, 2009.

[7] J. Lichtenberg, N. F. de Rooij, and E. Verpoorte, "Sample pretreatment on microfabricated devices," Talanta, vol. 56, no. 2, pp. 233-266, Feb. 2002.

[8] D. L. DeVoe and C. S. Lee, "Microfluidic technologies for MALDIMS in proteomics," Electrophoresis, vol. 27, no. 18, pp. 3559-3568, Sep. 2006.

[9] J. Bergkvist, S. Ekstrom, L. Wallman, M. Lofgren, G. Marko-Varga, J. Nilsson, and T. Laurell, "Improved chip design for integrated solid-phase microextraction in on-line proteomic sample preparation," Proteomics, vol. 2, no. 4, pp. 422-429, Apr. 2002.

[10] S. Ekstrom, L. Wallman, D. Hok, G. Marko-Varga, and T. Laurell, "Miniaturized solid-phase extraction and sample preparation for MALDI MS using a microfabricated integrated selective enrichment target," J. Proteome Res., vol. 5, no. 5, pp. 1071-1081, May 2006.

[11] W. Chen, J. Shen, X. Yin, and Y. Yu, "Optimization of microfabricated nanoliter-scale solid-phase-extraction device for detection of gel- separated proteins in low abundance by matrix-assisted laser desorption/ ionization mass spectrometry," Rapid Commun. Mass Spectrom., vol. 21, no. 1, pp. 35-43, Jan. 2007.

[12] E. A. S. Doherty, C.-W. Kan, B. M. Paegel, S. H. I. Yeung, S. Cao, R. A. Mathies, and A. E. Barron, "Sparsely cross-linked 'nanogel' matrixes as fluid, mechanically stabilized polymer networks for highthroughput microchannel DNA sequencing," Anal. Chem., vol. 76, no. 18, pp. 5249-5256, Sep. 2004.

[13] Y. Yang, C. Li, K. H. Lee, and H. G. Craighead, "Coupling on-chip solid-phase extraction to electrospray mass spectrometry through integrated electrospray tip," Electrophoresis, vol. 26, no. 19, pp. 3622-3630, Oct. 2005.

[14] L. Wallman, S. Ekstrom, G. Marko-Varga, T. Laurell, and J. Nilsson, "Autonomous protein sample processing on-chip using solid-phase microextraction, capillary force pumping, and microdispensing," Electrophoresis, vol. 25, no. 21, pp. 3778-3787, Nov. 2004.

[15] A. J. Handley, Extraction Methods in Organic Analysis, vol. 2, 1st ed. Boca Raton, FL: CRC Press, 1999.

[16] V. Meyer, Practical High-Performance Liquid Chromatography. Chichester, U.K.: Wiley, 1994.

[17] T. Nguyen, R. Pei, M. Stojanovic, and Q. Lin, "An aptamerbased microfluidic device for thermally controlled affinity extraction," Microfluidics Nanofluidics, vol. 6, no. 4, pp. 479-487, Apr. 2009.

[18] C. Mannironi, A. Di Nardo, P. Fruscoloni, and G. P. Tocchini-Valentini, "In vitro selection of dopamine RNA ligands," Biochemistry, vol. 36, no. 32, pp. 9726-9734, Aug. 1997.

[19] D. Nieuwlandt, M. Wecker, and L. Gold, "In-vitro selection of RNA ligands to substance-P," Biochemistry, vol. 34, no. 16, pp. 5651-5659, Apr. 1995.

[20] A. Geiger, P. Burgstaller, H. von der Eltz, A. Roeder, and M. Famulok, "RNA aptamers that bind L-arginine with sub-micromolar dissociation constants and high enantioselectivity," Nucleic Acids Res., vol. 24, no. 6, pp. 1029-1036, Mar. 1996.

[21] S. E. Lupold, B. J. Hicke, Y. Lin, and D. S. Coffey, "Identification and characterization of nuclease-stabilized RNA molecules that bind human prostate cancer cells via the prostate-specific membrane antigen," Cancer Res., vol. 62, no. 14 , pp. 4029-4033, Jul. 2002.

[22] W. James, "Aptamers in the virologists' toolkit," J. Gen. Virol., vol. 88, no. 2, pp. 351-364, Feb. 2007.

[23] R. S. Foote, J. Khandurina, S. C. Jacobson, and J. M. Ramsey, "Preconcentration of proteins on microfluidic devices using porous silica membranes," Anal. Chem., vol. 77, no. 1, pp. 57-63, Jan. 2005.

[24] C. Tuerk and L. Gold, "Systematic evolution of ligands by exponential enrichment: RNA ligands to bacteriophage T4 DNA polymerase," Science, vol. 249, no. 4968, pp. 505-510, Aug. 1990.

[25] P. Burgstaller, A. Girod, and M. Blind, "Aptamers as tools for target prioritization and lead identification," Drug Discov. Today, vol. 7, no. 24, pp. 1221-1228, Dec. 2002.

[26] L. S. Green, C. Bell, and N. Janjic, "Aptamers as reagents for highthroughput screening," Biotechniques, vol. 30, no. 5, p. 1094, May 2001.

[27] E. N. Brody, M. C. Willis, J. D. Smith, S. Jayasena, D. Zichi, and L. Gold, "The use of aptamers in large arrays for molecular diagnostics," Mol. Diagn., vol. 4, no. 4, pp. 381-388, Dec. 1999.

[28] S. M. Nimjee, C. P. Rusconi, R. A. Harrington, and B. A. Sullenger, "The potential of aptamers as anticoagulants," Trends Cardiovasc. Med., vol. 15, no. 1, pp. 41-45, Jan. 2005.

[29] C. Ravelet, C. Grosset, and E. Peyrin, "Liquid chromatography, electrochromatography, and capillary electrophoresis applications of DNA and RNA aptamers," J. Chromatogr. A, vol. 1117, no. 1, pp. 1-10, Jun. 2006.

[30] Y. Y. Feng, Z. Y. Zhou, X. Y. Ye, and H. J. Xiong, "Passive valves based on hydrophobic microfluidics," Sens. Actuators A, Phys., vol. 108, no. 1-3, pp. 138-143, Nov. 2003.

[31] C. Beldjord, M. Bornens, E. Bursaux, J. C. Dreyfus, P. Edery, A. Fischer, H. Gilgenkrantz, J. P. Grunfeld, A. Kahn, D. Labie, V. Lotteau, S. Lyonnet, A. Munnich, C. Nihoulfekete, and C. Derouffignac, "Muscle adenosinemonophosphate deaminase deficiency (AMPD1)," M S-Medecine Sci., vol. 9, pp. 983-984, 1993.

[32] R. Darby, Chemical Engineering Fluid Mechanics. New York: Marcel Dekker, 2001.

[33] J. P. Boulenger, N. Salem, P. J. Marangos, and T. W. Uhde, "Plasma adenosine levels-Measurement in humans and relationship to the anxiogenic effects of caffeine," Psychiatry Res., vol. 21, no. 3, pp. 247-255, Jul. 1987.

[34] T. H. Nguyen, C. Qiu, R. Pei, M. Stojanovic, J. Ju, and Q. Lin, “An integrated microfluidic system for affinity extraction and concentration of biomolecules coupled to MALDI-MS," in Proc. IEEE Int. Conf. MEMS, Tucson, AZ, 2008, pp. 196-199. 
[35] Y. D. Xu, M. L. Bruening, and J. T. Watson, "Non-specific, on-probe cleanup methods for MALDI-MS samples," Mass Spectrom. Rev., vol. 22, no. 6, pp. 429-440, Nov./Dec. 2003.

[36] I. P. Smirnov, X. Zhu, T. Taylor, Y. Huang, P. Ross, I. A. Papayanopoulos, S. A. Martin, and D. J. Pappin, "Suppression of alpha-cyano-4-hydroxycinnamic acid matrix clusters and reduction of chemical noise in MALDI-TOF mass spectrometry," Anal. Chem., vol. 76, no. 10, pp. 2958-2965, May 2004.

[37] S. Ekstrom, J. Malmstrom, L. Wallman, M. Lofgren, J. Nilsson, T. Laurell, and G. Marko-Varga, "On-chip microextraction for proteomic sample preparation of in-gel digests," Proteomics, vol. 2, no. 4, pp. 413-421, Apr. 2002.

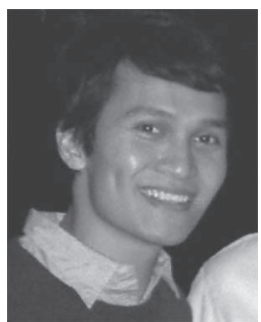

Thai Huu Nguyen received the B.Sc. degree in aerospace engineering in 2005 from the University of Virginia, Charlottesville, and the M.S. degree in mechanical engineering in 2007 from Columbia University, New York, NY, where he is currently working toward the Ph.D. degree in mechanical engineering.

His research interests include integrating aptamers in microfluidic platforms for biochemicaland biomedical-related applications.

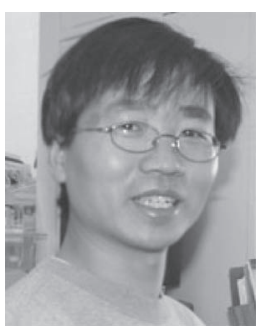

Renjun Pei received the B.Sc. and Ph.D. degrees from Wuhan University, Wuhan, China, in 1993 and 1998 , respectively.

From 2001 to 2003, he held a JSPS Postdoctoral Fellowship at the Institute for Chemical Research, Kyoto University, Kyoto, Japan. He is currently with the Department of Medicine, Columbia University, New York, NY. His research interests include aptamers, light-up probes, allosteric deoxyribozymes, molecular robotics, and bio/nanotechnologies.

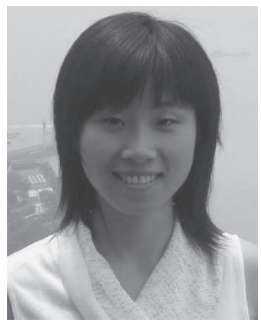

Chunmei Qiu received the B.Sc. degree in biological engineering in 2006 from Tianjin University, Tianjin, China, and the M.S. degree in chemical engineering in 2008 from Columbia University, New York, NY, where she is currently working toward the Ph.D. degree in genotyping by MALDI-TOF MS.

Her research focuses are on the development of next-generation DNA sequencing technology.

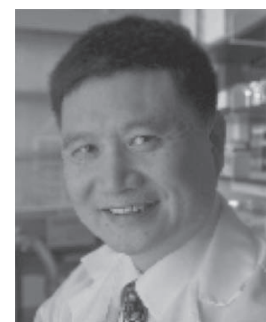

Jingyue Ju received the Ph.D. degree in bioorganic chemistry from the University of Southern California, Los Angeles, in 1993.

From 1993 to 1995 , he was a U.S. Department of Energy Human Genome Distinguished Postdoctoral Fellow at the University of California at Berkeley, where he coinvented the fluorescent energy-transfer labeling technology for DNA sequencing and analysis. From 1995 to 1999, he was a Senior Scientist and the Director of Chemistry and Assay Development with Incyte Genomics, Palo Alto, CA. Since 1999, he has been with Columbia University, New York, NY, where he is an Associate Professor of chemical engineering and the Head of DNA Sequencing and Chemical Biology at the Columbia Genome Center. His research interests are in the design and synthesis of new molecular tags for biological labeling and imaging, and developing new technologies for genomic research.

Dr. Ju was the recipient of a Packard Fellowship in science and engineering.

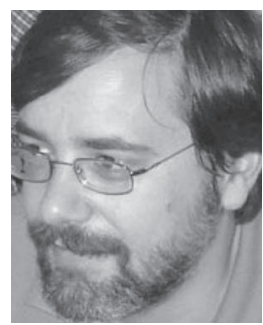

Milan Stojanovic received the B.Sc. degree from Beogradski Univerzitet, Belgrade, Serbia, and the $\mathrm{Ph} . \mathrm{D}$. degree in organic chemistry from Harvard University, Cambridge, MA.

He was a Postdoctoral Fellow and is currently a faculty member in the Department of Medicine, Columbia University, New York, NY.

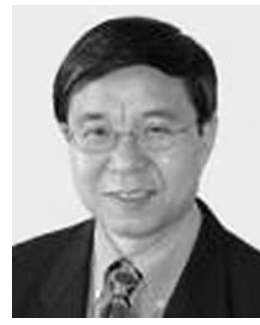

Qiao Lin received the Ph.D. degree in mechanical engineering from the California Institute of Technology (Caltech), Pasadena, in 1998. His Ph.D. thesis concerned robotics.

From 1998 to 2000, he conducted postdoctoral research in microelectromechanical systems (MEMS) at the Caltech Micromachining Laboratory. From 2000 to 2005, he was an Assistant Professor of mechanical engineering at Carnegie Mellon University, Pittsburgh, PA. Since 2005, he has been an Associate Professor of mechanical engineering at Columbia University, New York, NY. His research interests are in designing and creating integrated micro/nanosystems, in particular MEMS and microfluidic systems, for biomedical applications. 